\title{
Optical fine-needle aspiration biopsy in a rat model
}

Dremin, Viktor, Potapova, Elena, Zherebtsov, Evgeny, Kozlov, Igor, Seryogina, Evgeniya, et al.

Viktor Dremin, Elena Potapova, Evgeny Zherebtsov, Igor Kozlov, Evgeniya Seryogina, Ksenia Kandurova, Alexander Alekseyev, Gennadii Piavchenko, Sergey Kuznetsov, Andrian Mamoshin, Andrey Dunaev, "Optical fine-needle aspiration biopsy in a rat model," Proc. SPIE 10877, Dynamics and

Fluctuations in Biomedical Photonics XVI, 108770K (1 March 2019); doi: $10.1117 / 12.2509255$

SPIE. Event: SPIE BiOS, 2019, San Francisco, California, United States 


\title{
Optical fine-needle aspiration biopsy in a rat model
}

\author{
Viktor Dremin ${ }^{\mathrm{a}}$, Elena Potapova ${ }^{\mathrm{a}}$, Evgeny Zherebtsov $^{\mathrm{a}}$, Igor Kozlov ${ }^{\mathrm{a}}$, Evgeniya Seryogina ${ }^{\mathrm{a}}$, \\ Ksenia Kandurova $^{\mathrm{a}}$, Alexander Alekseyev ${ }^{\mathrm{b}}$, Gennadii Piavchenko ${ }^{\mathrm{c}, \mathrm{d}}$, Sergey Kuznetsov ${ }^{\mathrm{c}}$, \\ Andrian Mamoshin ${ }^{\mathrm{a}, \mathrm{e}}$, and Andrey Dunaev ${ }^{\mathrm{a}}$ \\ ${ }^{a}$ Research Development Center of Biomedical Photonics, Orel State University, \\ Komsomolskaya Str. 95, Orel, Russia, 302026 \\ ${ }^{\mathrm{b}}$ Department of Anatomy, Operative Surgery and Emergency Medicine, Medical Institute, Orel \\ State University, 95 Komsomolskaya St., Orel, Russia, 302026 \\ ${ }^{\mathrm{c} H i s t o l o g y, ~ C y t o l o g y}$ and Embryology Department, I.M. Sechenov First Moscow State Medical \\ University (Sechenov University), Mokhovaya Str. 11, Moscow, Russia, 125009 \\ ${ }^{\mathrm{d}}$ Centre of Preclinical Research, JSC "Retinoids", Plekhanova 2/46-5, Moscow, Russia, 111123 \\ ${ }^{e}$ Orel Regional Clinical Hospital, Orel, Russia, 302028
}

\begin{abstract}
Fine needle aspiration biopsy technique and following histological examination show its effectiveness and safety but its performing takes several time. However, the problem of real-time analysis of pathological changes in tissues remains relevant. We demonstrate optical fine-needle biopsy method, combining a fine needle (17.5G) and a fiber-optic probe (1 mm diameter) for minimally invasive interrogation of tissue in vivo. During rat tumor experiment, we collected spectrally-resolved diffuse reflectance and fluorescence. Quantified differences between tumor and normal tissues were demonstrated and approved with morphological analysis. The proposed methodology seems promising for developing new diagnostic criteria for clinical practice.
\end{abstract}

Keywords: optical biopsy, fluorescence spectroscopy, diffuse reflectance spectroscopy, fine-needle aspiration biopsy, tumor, minimally invasive surgery

\section{INTRODUCTION}

In recent decades, there has been an increase in patients with malignant tumors of the abdominal organs. In particular, hepatocellular carcinoma remains one of the most common malignant tumors and leading cause of cancer-related deaths worldwide. ${ }^{1}$ However, there are no sufficiently accurate diagnostic methods that allow for clarifying the nature of pathological formation at the stage of obtaining a biopsy material in vivo and in situ. The biopsy taken at the time of surgery operation demands immediate analysis. Otherwise, it necessitates the repeated surgical intervention which usually leads to a worsening of the prognosis of the disease. Development of efficient technology for optical biopsy may offer a solution of the problem. ${ }^{2,3}$ The ability to recognize the suspect tissue in a short time without interruption of the operation is able to increase the effectiveness of the endoscopic surgery of the cancerous tumors.

Currently, many researchers apply the method of fluorescence spectroscopy (FS) for studies of tissue or cellular metabolism. ${ }^{4,5}$ The FS application in oncology is based on differences in the intensity and spectral composition of the fluorescence of healthy and malignant tissues when excited by laser radiation in the UV or visible range of the spectrum. ${ }^{6}$ Another technique named diffuse reflectance spectroscopy (DRS) provides information about light absorption in tissues. DRS is used separately or in combination with FS. This method allows to differentiate malignant and benign tumors as well, as neoplastic tissue undergo significant architectural changes at the cellular and intracellular levels. ${ }^{7}$ The combination of several complementary optical modalities can give more valuable information for diagnosis and treatment.

Send correspondence to Viktor Dremin

E-mail: dremin_viktor@mail.ru,

Telephone: +79536127713

Dynamics and Fluctuations in Biomedical Photonics XVI, edited by Valery V. Tuchin, Martin J. Leahy, Ruikang K. Wang Proc. of SPIE Vol. 10877, 108770K · C 2019 SPIE · CCC code: 1605-7422/19/\$18 · doi: 10.1117/12.2509255 


\section{MATERIAL AND METHODS}

In this study, we present experimental studies with a fine-needle fiber optic probe comprising emitting and collecting fibers for measurements of fluorescence intensity (with excitation at $365 \mathrm{~nm}$ and $450 \mathrm{~nm}$ ) and diffuse reflectance (in the range 400-900 $\mathrm{nm}$ ).

The choice of wavelengths of FS channel is caused by NADH and FAD coenzymes and collagen fluorescence excitation. The DRS channel includes HL-2000-FHSA Tungsten Halogen Light Source ("Ocean Optics", USA) with a wavelength range of $360-2400 \mathrm{~nm}$. The light collected from the tissue is filtered by optical filter to attenuate backscattered radiation and then it is analyzed by a CCD Flame Spectrometer ("Ocean Optics", USA) in the range of 350-1000 nm (Fig.1a). System control and further data processing is performed using a personal computer with custom developed software in Matlab program environment.

The optic probe has been developed to be compatible with the $17.5 \mathrm{G}$ biopsy needle standard and has the diameter of $1 \mathrm{~mm}$. The probe has 10 optical fibers. The nine transmitting ones (100 $\mu \mathrm{m}$ each) include three fibers connected to a halogen light source, three fibers connected to a laser diode and three fibers connected to an LED. The fibers are located around the central one $(200 \mu \mathrm{m})$, which delivers the collected light to spectrometer. The amount and orientation of optical fibers inside the fiber-optic probe provides uniform and bright illumination of the diagnostic volume and allows reaching a high signal-to-noise ratio. The probe has an bevel angle of 20 degrees, which ensures reliable contact of the probe with dense tissues.

\section{RESULTS AND DISCUSSION}

With the probe, measurements of pathologically altered rat tissues of the in vivo were carried out. The animal was stored in the Center of Preclinical Research in accordance with GLP principles. The studies have been combined with the standard procedure of the biopsy sampling (Fig.1b). After the spectroscopy analysis the tumor tissue was fixed in the neutral formalin and embedded in parafin. The $5 \mu \mathrm{m}$ sections were stained by haematoxylin and eosin method with a standard operational procedures.
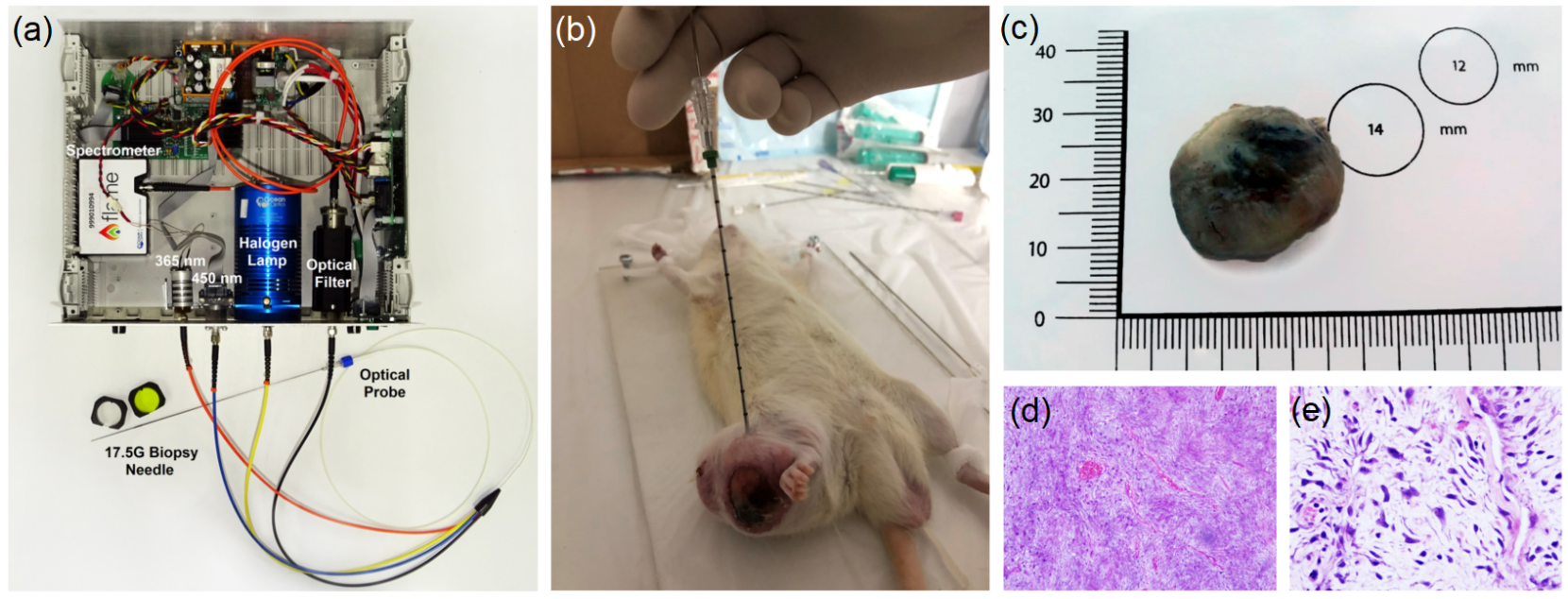

Figure 1. (a) The multimodal FS/DRS fine needle setup; (b) rat tumor experiment; (c) fragment of a tumor node up to $2.5 \mathrm{~cm}$ in a diameter; histological examination of tumor tissue with haematoxylin and eosin staining at (d) x100 and (e) $\mathrm{x} 400$.

The morphological picture (Fig.1d,e) of tumor corresponds to low-grade myxofibrosarcoma 8811/3. Fig.2a,b show that for rat tissues measured in vivo, there are significant differences between fluorescence spectra of intact tissue and myxofibrosarcoma. This may be due to changes in NADH, FAD and collagen content. Fig.2c shows that for rat tissues, there are significant differences between the diffuse reflectance spectra of normal and tumor tissue, notably in the wavelength range 400-600 nm. Reflectance measurements clearly show the pronounced reflectance peak in region 450-500 $\mathrm{nm}$. Interesting is the absence of peaks of oxyhemoglobin absorption on the tumor spectra, which indicates tissue ischemia. 

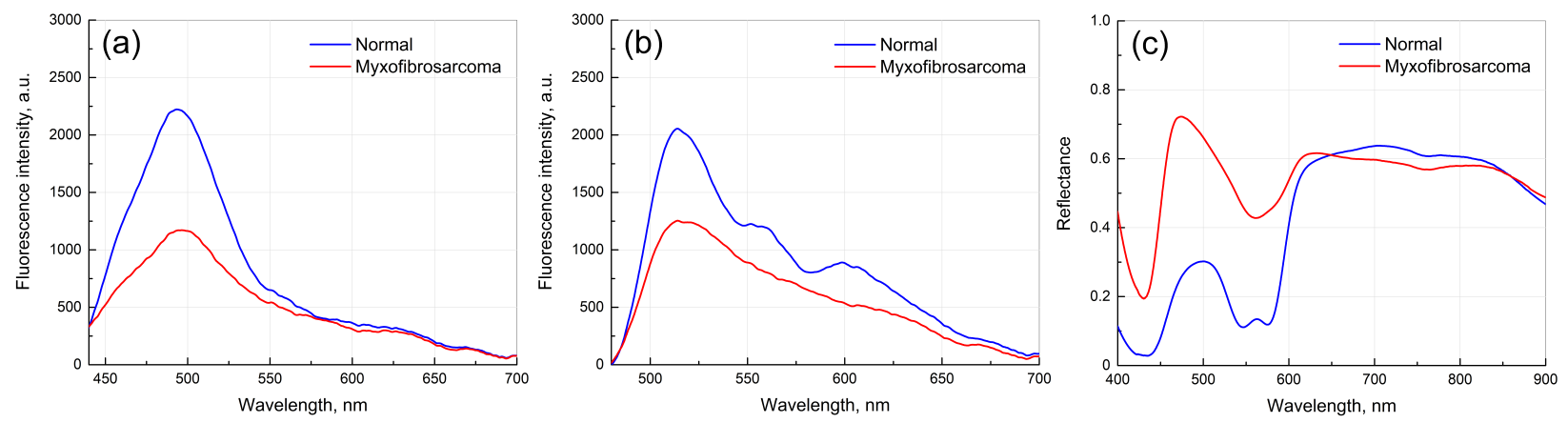

Figure 2. Fluorescence spectra at excitation wavelengths (a) $365 \mathrm{~nm}$, (b) $450 \mathrm{~nm}$ and (c) diffuse reflectance spectra of normal (blue line) and tumor rat tissue (red line).

\section{CONCLUSION}

From optical measurements acquired during rat tumor experiment, we verified the ability to collect spectrallyresolved diffuse reflectance and fluorescence rapidly in vivo. Overall, these results suggest that optical fine-needle aspiration biopsy is a promising method for the improved diagnosis of tumor in vivo.

Simultaneous registration of fluorescence and diffuse reflectance spectra carries the information about the corresponding parameters of metabolic processes and morphological structure of tissues, which is of interest for clinical practice. As the biopsy remains a valuable diagnostic method required to ensure the diagnosis, the necessity of real-time analysis determine the relevance of optical biopsy research. The proposed methodology of acquiring the optical parameters data and its correspondence with biopsy samples from almost the same diagnostic volume will provide the basis for development of automatic classifier using the neural network. This will make diagnostics during minimally invasive surgical procedures faster and accessible, will increase its accuracy and reliability.

\section{ACKNOWLEDGMENTS}

This study was supported by the Russian Science Foundation under project No.18-15-00201.

\section{REFERENCES}

[1] Clark, T., Maximin, S., Meier, J., Pokharel, S., and Bhargava, P., "Hepatocellular carcinoma: Review of epidemiology, screening, imaging diagnosis, response assessment, and treatment," Current Problems in Diagnostic Radiology 44(6), 479-486 (2015).

[2] Kandurova, K., Dremin, V., Zherebtsov, E., Potapova, E., Dunaev, A., Mamoshin, A., Alyanov, A., and Muradyan, V., "Application of the fluorescence spectroscopy for the analysis of the state of abdominal cavity organs tissues in mini-invasive surgery," Proc. SPIE 10685, 106854P (2018).

[3] Kandurova, K., Dremin, V., Zherebtsov, E., Potapova, E., Alyanov, A., Mamoshin, A., Ivanov, Y., Borsukov, A., and Dunaev, A., "Fiber-optic system for intraoperative study of abdominal organs during minimally invasive surgical interventions," Applied Sciences 9(2) (2019).

[4] Walsh, A., Cook, R. S., Rexer, B., Arteaga, C. L., and Skala, M. C., "Optical imaging of metabolism in her2 overexpressing breast cancer cells," Biomed. Opt. Express 3(1), 75-85 (2012).

[5] Bartolome, F. and Abramov, A. Y., "Measurement of mitochondrial nadh and fad autofluorescence in live cells.," Methods in molecular biology 1264, 263-70 (2015).

[6] Rafailov, I., Palmer, S., Litvinova, K., Dremin, V., Dunaev, A., and Nabi, G., "A novel excitation-emission wavelength model to facilitate the diagnosis of urinary bladder diseases," Proc. SPIE 9303, 93030W (2015).

[7] Lloyd, W. R., Wilson, R. H., Lee, S. Y., Chandra, M., McKenna, B., Simeone, D., Scheiman, J., and Mycek, M.-A., "In vivo optical spectroscopy for improved detection of pancreatic adenocarcinoma: a feasibility study," Biomed. Opt. Express 5(1), 9-15 (2014). 